\title{
Sedated gastroscopy improves detection of gastric polyps
}

\author{
MENG-JUN CHEN, WEI WU, SHUANG PAN, CHUN-JING LIN, LE-MEI DONG, \\ ZHOU-FENG CHEN, JIAN-SHENG WU and ZHI-MING HUANG
}

\begin{abstract}
Department of Gastroenterology, The First Affiliated Hospital of Wenzhou Medical University, Wenzhou, Zhejiang 325000, P.R. China
\end{abstract}

Received December 3, 2017; Accepted April 16, 2018

DOI: $10.3892 /$ etm.2018.6525

\begin{abstract}
The detection rate of gastric polyps (GPs) is low, improving the detection rate would be good. The present study aimed to evaluate the role of sedated gastroscopy in GP detection. The data of patients who underwent gastroscopic examination from January 2014 to December 2016 at the First Affiliated Hospital of Wenzhou Medical University (Wenzhou, China) were retrospectively reviewed. Endoscopic records of 6,195 patients diagnosed with GPs were analyzed. The GP detection rate was 3.12 and $5.11 \%$ in the unsedated and sedated gastroscopy group, respectively $(\mathrm{P}<0.05)$. Also after stratification by sex, the GP detection rate was significantly higher in the sedated gastroscopy group $(\mathrm{P}<0.05)$. In addition, patients aged $\geq 20$ years in the sedated gastroscopy group had a higher GP detection rate than those in the unsedated gastroscopy group $(\mathrm{P}<0.05)$. The incidence of cardiac, gastric fundus, gastric body and multiple-site GPs was significantly different between the two groups $(\mathrm{P}<0.05)$. GPs $\leq 0.5$ and $>0.5 \mathrm{~cm}$ were more common in the sedated gastroscopy group than in the unsedated gastroscopy group $(\mathrm{P}<0.05)$. The common pathologic types of GPs were gastric fundus gland (52.27\%) and hyperplastic polyps (34.74\%). In conclusion, the GP detection rate may be improved by inhibition of gastric muscle cramping with sedation.
\end{abstract}

\section{Introduction}

Gastric polyps (GPs) are defined as protuberant mucosal lesions that are usually detected endoscopically. Most patients are asymptomatic; however, depending on their size and location, some large GPs may cause symptoms, including bleeding, chest pain, abdominal pain, anemia and even gastric tract obstruction (1-4).

Correspondence to: Professor Jian-Sheng Wu, Department of Gastroenterology, The First Affiliated Hospital of Wenzhou Medical University, 12 Nanbaixiang Road, Ouhai, Wenzhou, Zhejiang 325000, P.R. China

E-mail:wzwujs@163.com

Key words: gastroscopy, sedated, gastric polyps, detection rate
The widespread use of gastroscopy in China contributes to the improvement of the detection rate of GPs. Previous studies have reported that the rate of GPs was between 1.0-6.4\% and the prevalence of GPs has been gradually increasing over the past decade (4-6). In addition, GPs were less common in males than in females, with a ratio of 1:1.8 to 1:2.5 (4-6). Other studies also indicated that the detection rate of GPs increases with increasing age $(7,8)$. The most common pathologic type of GPs are gastric fundus gland polyp and hyperplastic polyp, which are associated with an increased risk of gastric cancer $(7,8)$. Thus, it is important to further improve the detection rate of GPs.

Sedated gastroscopy, i.e., gastroscopy under conscious sedation, improves the patients' tolerance of procedures and in recent years, it has become increasingly popular in China. The present study hypothesized that the GP detection rate on gastroscopy may be improved with sedation. Thus, the aim of this retrospective observational study was to assess the superiority of sedated gastroscopy over unsedated gastroscopy in detecting GPs.

\section{Patients and methods}

Patients. In the present retrospective study, patients who underwent gastroscopy with gastrointestinal symptoms, including abdominal distention, abdominal pain, belching and sour regurgitation or without symptoms at the First Affiliated Hospital of Wenzhou Medical University (Wenzhou, China) between January 1,2014 and December 31, 2016 were included. Patients decided to undergo gastroscopic examination with or without sedation by themselves, after having been informed about the benefits, risks and limitations of sedation, as well as those of unsedated gastroscopy, according to the recommendations of the American Society for Gastrointestinal Endoscopy (9). No additional procedures were performed during the gastroscopic examination. Those patients with GPs diagnosed under gastroscopy were recorded, and the pathologies were confirmed as inflammatory polyp, hyperplastic polyp, gastric fundus gland polyp adenomas, juvenile polyp, squamous hyperplasia and adenocarcinoma, while pathologies confirmed as pathology-negative polyp, xanthelasma, mesenchymoma, lymphoma, leiomyoma, ectopic pancreas, carcinoid and hamartoma were excluded within 7 working days by experienced pathologists. In addition, patients who had a history of gastric cancer, colon cancer, stomach surgery 
or colon surgery were excluded. Patients who met the exclusion criteria were deleted when detection rate was calculated. A total of 165,142 patients were included in the current study. A total of 112,512 patients underwent unsedated gastroscopy, whereas 52,630 patients underwent sedated gastroscopy (propofol, $2 \mathrm{mg} / \mathrm{kg}$, intravenous) with monitoring via electrocardiograms.

Data. The present study retrospectively analyzed the data of 165,142 patients who underwent gastroscopy, and 6,195 patients with GPs were diagnosed on gastroscopy. The patient data were obtained from endoscopy and pathologic reports, with parameters extracted including sex, age, inspection time and GP characteristics (location, histological diagnosis, number and size).

Statistical analysis. Data were analyzed using SPSS (version 17.0; SPSS, Inc., Chicago, IL, USA). Patient age was expressed as the mean \pm standard deviation. The detection rate in the two groups was standardized prior to the $\mathrm{c}^{2}$ test due to a disproportionate age distribution. $\mathrm{P}<0.05$ was considered to indicate a statistically significant difference.

\section{Results}

Patient characteristics and procedure time are not different. A total of 165,142 gastroscopies were performed within 3 years at the Digestive Endoscopy unit of the First Affiliated Hospital of Wenzhou Medical University (Wenzhou, China). No statistically significant difference in the sex distribution and procedure time were identified between the two groups (Table I).

Comparison of GP detection rate between sedated and unsedated gastroscopy group. A total of 6,195 GPs were diagnosed by gastroscopy and pathologic examination. Patients were stratified into 5 different groups based on age. The detection rate significantly differed between the unsedated and sedated gastroscopy groups of patients aged 20-39, 40-59, $60-79$ and $\geq 80$ years, but not of those aged $<20$ years (Table II). Considering the age distribution, the GP detection rate of these two groups was standardized prior to the statistical analysis, and the result indicated a significantly higher detection rate in the sedated gastroscopy group compared with that in the unsedated gastroscopy group (Table III).

The present analysis indicated that the rate of GP detection in female patients was significantly higher than that in male patients regardless of sedation $(\mathrm{P}=0.001)$. In addition, the GP detection rates for male and female patients receiving sedated gastroscopy were significantly higher than for those receiving unsedated gastroscopy (Table II).

The location of GPs between the unsedated and sedated gastroscopy groups included cardiac, gastric fundus, gastric body and multiple sites, which means that GPs were detected in $\geq 2$ anatomy sections of the stomach. The incidence of cardiac, gastric fundus, gastric body and multiple-site GPs was significantly different between the unsedated and sedated gastroscopy groups, due to the different diagnostic performance of sedated and unsedated gastroscopy (Figs. 1 and 2). Whereas the incidence of gastric angular and gastric antrum
Table I. Sex distribution and procedure time in the two groups.

\begin{tabular}{lcc}
\hline Parameter & $\begin{array}{c}\text { Unsedated gastroscopy } \\
\text { group }(112,512)\end{array}$ & $\begin{array}{c}\text { Sedated gastroscopy } \\
\text { group }(52,630)\end{array}$ \\
\hline Sex & & \\
Male & $60,880(54.11 \%)$ & $28,108(53.41 \%)$ \\
Female & $51,632(45.89 \%)$ & $24,522(46.59 \%)$ \\
Age (years) & $51.90 \pm 13.73$ & $49.67 \pm 12.23$ \\
Procedure & $321.2 \pm 97.5$ & $305.1 \pm 90.4$ \\
time (sec) & & \\
\hline
\end{tabular}

Values are expressed as $\mathrm{n}$ or the mean \pm standard deviation or number $(\%)$. There were no significant differences between the groups.

polyps was not significantly different between these two groups (Table IV).

Pathological characteristics of GPs. As presented in Table IV, the standardized GP detection rates in the sedated gastroscopy group with lesion diameters of $\leq 0.5$ and $>0.5 \mathrm{~cm}$ were significantly higher than those in the unsedated gastroscopy group.

Pathologic examination of tissue samples of the 6,195 GPs identified them as gastric fundus gland polyp $(n=3,238)$, hyperplastic polyp $(n=2,152)$, inflammatory polyp $(n=531)$, squamous hyperplasia $(n=103)$, adenomatous polyp $(n=116)$, adenocarcinoma $(n=35)$ and juvenile polyp $(n=20)($ Table V).

\section{Discussion}

GPs are usually detected endoscopically. It has been reported that $\sim 11 \%$ of gastric adenomatous polyps developed into carcinoma in situ within 4 years of follow-up (10). After excision, the recurrence rate is $16 \%$ (6). At present, no precise and relevant epidemiological data on GPs are available.

In the present study, the overall detection rate of GPs was $3.75 \%$, which is in accordance with the results of previous studies (4-6). The patients were stratified into an unsedated gastroscopy group and a sedated gastroscopy group. No statistically significant difference in the procedure time was present between these two groups, but a disproportion regarding patient age was encountered. A lower proportion of patients aged $\geq 60$ years underwent sedated gastroscopy, which may be attributed to cautious anesthesia evaluation. Thus, the GP detection rate of these two groups was standardized prior to statistical analysis. The GP detection rate was higher in the sedated gastroscopy group than that in the unsedated gastroscopy group, and the difference was statistically significant, which implies that GPs may be more easily detected under sedated gastroscopy. Furthermore, the present study suggested that patients aged $\geq 20$ years have a significantly higher GP detection rate under sedated gastroscopy. In patients aged $<20$ years, GPs were more often detected in the unsedated gastroscopy group than in the sedated gastroscopy group, but the difference was not statistically significant. This may be due to the limited sample size.

The present study assessed and quantified the location of the GPs, and the results indicated that the detection rates of 
Table II. Clinicopathological characteristics of GP patients.

\begin{tabular}{|c|c|c|c|c|}
\hline \multirow[b]{2}{*}{ Feature } & \multicolumn{2}{|c|}{ GP detection rate, $\%(\mathrm{n} / \mathrm{m})$} & \multirow[b]{2}{*}{$\chi^{2}$} & \multirow[b]{2}{*}{ P-value } \\
\hline & Unsedated gastroscopy group & Sedated gastroscopy group & & \\
\hline \multicolumn{5}{|l|}{ Age (years) } \\
\hline$<20^{\mathrm{a}}$ & $0.97(5 / 515)$ & $1.32(2 / 151)$ & & 0.66 \\
\hline $20-39$ & $2.00(410 / 20,482)$ & $3.54(387 / 10,945)$ & 67.9 & 0.001 \\
\hline $40-59$ & $3.06(1,754 / 57,301)$ & $5.19(1,538 / 29,606)$ & 243.9 & 0.001 \\
\hline $60-79$ & $3.95(1,247 / 31,536)$ & $6.41(740 / 11,538)$ & 116.1 & 0.001 \\
\hline$\geq 80$ & $3.32(89 / 2,678)$ & $5.90(23 / 390)$ & 6.4 & 0.011 \\
\hline \multicolumn{5}{|l|}{ Sex } \\
\hline Male & $2.11(1,284 / 60,880)$ & $3.86(1,086 / 28,108)$ & 228.4 & 0.001 \\
\hline Female & $4.30(2,221 / 51,632)$ & $6.54(1,604 / 24,522)$ & 174.8 & 0.001 \\
\hline \multicolumn{5}{|l|}{ GP location } \\
\hline Cardiac & $0.35(398 / 112,512)$ & $0.46(242 / 52,630)$ & 22.9 & 0.001 \\
\hline Gastric fundus & $0.49(552 / 112,512)$ & $0.93(487 / 52,630)$ & 222.1 & 0.001 \\
\hline Gastric body & $1.15(1,297 / 11,2512)$ & $2.28(1,201 / 52,630)$ & 623.3 & 0.001 \\
\hline Gastric angular & $0.81(910 / 112,512)$ & $0.85(445 / 52,630)$ & 1.4 & 0.230 \\
\hline Gastric antrum & $0.07(79 / 112,512)$ & $0.08(43 / 52,630)$ & 1.3 & 0.249 \\
\hline Multiple-sites & $0.24(268 / 112,512)$ & $0.52(272 / 52,630)$ & 170.5 & 0.001 \\
\hline \multicolumn{5}{|c|}{ Diameter of GPs $(\mathrm{cm})$} \\
\hline$\leq 0.5$ & $2.75(3,089 / 112,512)$ & $4.57(2,405 / 52,630)$ & 779.5 & 0.001 \\
\hline$>0.5$ & $0.37(416 / 112,512)$ & $0.54(285 / 52,630)$ & 53.5 & 0.0001 \\
\hline
\end{tabular}

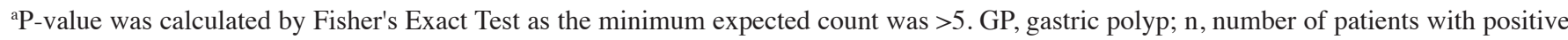
detection; $\mathrm{m}$, number of patients examined.

Table III. Standardized calculation of GP detection rate.

\section{Unsedated gastroscopy group}

Sedated gastroscopy group

Detection rate (\%) Expected GPs (n) Detection rate (\%) Expected GPs (n)

\begin{tabular}{lccccc} 
Age group (years) & Standard population $^{\mathrm{a}}$ & Detection rate $(\%)$ & Expected GPs $(\mathrm{n})$ & Detection rate $(\%)^{\text {Expected GPs }(\mathrm{n})}$ \\
\hline$<20$ & 666 & 0.97 & 6 & 1.32 & 9 \\
$20-39$ & 31,427 & 2.00 & 629 & 3.54 & 1,113 \\
$40-59$ & 86,907 & 3.06 & 2,659 & 5.19 & 4,510 \\
$60-79$ & 43,074 & 3.95 & 1,701 & 6.41 & 2,761 \\
$\geq 80$ & 3,068 & 3.32 & 102 & 5.90 & 181 \\
Total $^{\mathrm{b}}$ & 165,142 & & $5,097(3.09 \%)$ & $8,574(5.19 \%)$
\end{tabular}

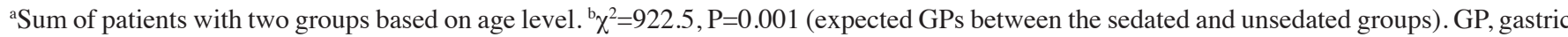
polyp.

cardiac, gastric fundus, gastric body and multiple-site GPs in the sedated gastroscopy group were statistically higher than those in the unsedated gastroscopy group. The poorer diagnostic performance of unsedated gastroscopy may be attributed to the following: i) Patients always vomit when undergoing unsedated gastroscopy, which causes shaking of the video screen; ii) vomiting causes the gastric muscle to shrink, making it inconvenient for the clinicians to observe each part of the gastral cavity, particularly the side of the gastric body with the greater curvature, which has numerous mucosal folds (Fig. 1); iii) drastic gastric muscle shrinking may cause mucosal hyperemia and edema during unsedated gastroscopy within a short time, which changes the base color of the gastric mucosa (Fig. 2). These factors may affect the diagnosis of GPs.

In the present study, a slight difference in the incidence of gastric angular polyps and gastric antrum polyps was identified between the two groups, but with no statistically significant difference. This is in accord with the actual situation, as the gastric smooth muscles of the antrum do not shrink as severely 
Table IV. Standardized calculation of GP location and size.

\begin{tabular}{|c|c|c|c|c|c|c|}
\hline \multirow[b]{2}{*}{ GP location } & \multicolumn{2}{|c|}{ Unsedated gastroscopy group } & \multicolumn{2}{|c|}{ Sedated gastroscopy group } & \multirow[b]{2}{*}{$\chi^{2}$} & \multirow[b]{2}{*}{ P-value } \\
\hline & Detection rate $(\%)$ & Expected GPs (n) & Detection rate $(\%)$ & Expected GPs (n) & & \\
\hline Cardiac & 0.35 & 584 & 0.46 & 759 & 22.9 & 0.001 \\
\hline Gastric fundus & 0.49 & 810 & 0.93 & 1,528 & 222.1 & 0.001 \\
\hline Gastric body & 1.15 & 1,904 & 2.28 & 3,768 & 623.3 & 0.001 \\
\hline Gastric antrum & 0.81 & 1,336 & 0.85 & 1,396 & 1.3 & 0.249 \\
\hline Gastric angular & 0.07 & 116 & 0.08 & 135 & 1.4 & 0.230 \\
\hline Multiple-site & 0.24 & 393 & 0.52 & 853 & 170.5 & 0.001 \\
\hline \multicolumn{7}{|l|}{ Size of GP $(\mathrm{cm})$} \\
\hline$\leq 0.5$ & 2.75 & 4,534 & 4.57 & 7,546 & 779.5 & 0.001 \\
\hline$>0.5$ & 0.37 & 611 & 0.54 & 894 & 53.5 & 0.001 \\
\hline
\end{tabular}

GP, gastric polyp.

Table V. Pathology type of GPs.

\begin{tabular}{lccr}
\hline Pathological type of GP & Unsedated gastroscopy group, $\mathrm{n}(\%)$ & Sedated gastroscopy group, $(\%)$ & Total, $\mathrm{n}(\%)$ \\
\hline Gastric fundus gland polyp & $1,282(20.69)$ & $1,956(31.57)$ & $3,238(52.27)$ \\
Hyperplastic polyp & $1,020(16.46)$ & $1,132(18.27)$ & $2,152(34.74)$ \\
Inflammatory polyp & $253(4.08)$ & $278(4.49)$ & $531(8.57)$ \\
Adenomatous polyp & $52(0.84)$ & $64(1.03)$ & $10(0.16)$ \\
Adenocarcinoma & $25(0.40)$ & $12(0.19)$ & $35(0.56)$ \\
Juvenile polyp & $8(0.13)$ & $53(0.86)$ & $1.87)$ \\
Squamous hyperplasia & $50(0.81)$ & & $103(1.66)$ \\
\hline
\end{tabular}

GP, gastric polyp.
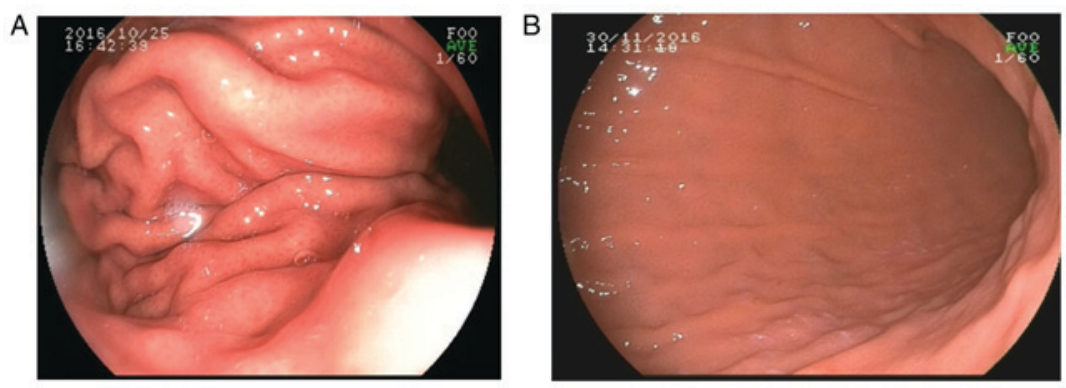

Figure 1. (A) Vomiting causes the gastric muscle to shrink during unsedated gastroscopy. (B) Relaxation of the gastric muscle during sedated gastroscopy.
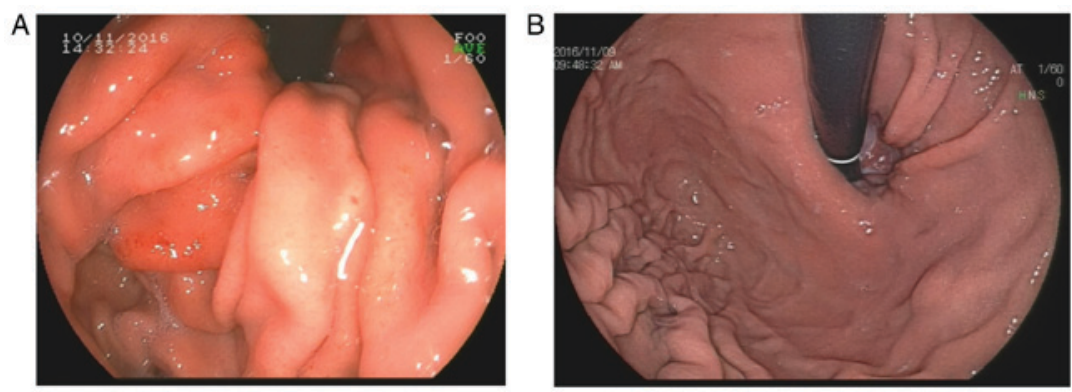

Figure 2. (A) Drastic gastric muscle shrinking causes mucosal hyperemia and edema during unsedated gastroscopy. (B) Normal-appearing gastric mucosa of gastric fundus during sedated gastroscopy. 
as those of the gastric body and are easier to expand by gas injection. Furthermore, the limited sample size of gastric angular polyps in the two groups may have affected the statistical analysis. As the current study was a single-center study, it was limited. Studies with larger samples sizes, may be able to demonstrate that the GP detection rate can be improved by sedated gastroscopy. More studies should be performed to further confirm the diagnostic advantage of sedated gastroscopy.

A study has reported that the predilection site of GPs was the gastric antrum (5). In another study, the gastric body has been reported to be the most common location (4). In the present study, the most common predilection site of polyps in the two groups was the gastric body. However, the second most common predilection sites were the gastric antrum in the unsedated gastroscopy group and the gastric fundus in the sedated gastroscopy group. The third most common predilection sites were the gastric fundus in the unsedated gastroscopy group and the gastric antrum in the sedated gastroscopy group. Therefore, it is implied that sedation not only makes patients comfortable during gastroscopy, but it will also make it easier for doctors to observe the gastric body and gastric fundus.

Various studies have reported on the common pathologic types of GP. Evans et al (10) reported that the most common pathologic type of GPs was the gastric fundus gland polyp, whereas other studies reported inflammatory polyp or hyperplastic polyp to be the most common pathologic types $(5,11)$. The present study indicated that the most common pathologic type of GPs was gastric fundus gland polyp (52.27\%), followed by hyperplastic $(34.74 \%)$ and inflammatory polyps $(8.57 \%)$. Sections from biopsies of the cardiac polyps were diagnosed as squamous epithelial hyperplasia, which may be due to the proliferation and migration of squamous epithelia to the cardiac site. Inaccurate positioning of biopsy to contain parts of the esophageal mucosal epithelium maybe another reason. Certain lesions were confirmed as adenomatous polyp (1.87\%), adenocarcinoma $(0.56 \%)$, and juvenile polyp $(0.32 \%)$, which require polypectomy or surgical treatment as soon as possible.

In conclusion, the results of the present study indicated that sedation enhances the diagnostic yield of GPs on gastroscopy. Considering its low cost and convenient application in South China, sedated gastroscopy is a better diagnostic option than unsedated gastroscopy.

\section{Acknowledgements}

Not applicable.

\section{Funding}

No funding received.

\section{Availability of data and materials}

The analyzed data sets generated during the study are available from the corresponding author on reasonable request.

\section{Authors' contributions}

MJC and J-SW conceived and designed the study. WW, SP, $\mathrm{C}-\mathrm{JL}$ and L-MD were involved in the acquisition, analysis and interpretation of data and drafting of the manuscript. $\mathrm{Z}-\mathrm{FC}, \mathrm{J}-\mathrm{SW}$ and Z-MH performed a critical revision of the manuscript and were responsible for the intellectual content, statistical analysis, supporting material and study supervision. The final version of the manuscript has been read and approved by all authors, and each author believes that the manuscript represents honest work.

\section{Ethics approval and consent to participate}

Not required due to the retrospective nature of the study.

\section{Patient consent for publication}

Not applicable.

\section{Competing interests}

The authors declare that they have no competing interests.

\section{References}

1. Nayudu SK, Niazi M, Balar B and Kumbum K: A rare complication of hyperplastic gastric polyp. Case Rep Gastrointest Med 2013: 631975, 2013.

2. He HY, Shen ZB, Fang Y, Sun YH and Qin XY: Bleeding and hyperpyrexia in an adult with gastric inflammatory fibroid polyp. Chin Med J (Engl) 126: 2594, 2013.

3. Vlacancich R, Sultan M and Al-Kawas F: Gastric outlet obstruction caused by prolapsed gastric polyp into the pyloric channel. Clin Gastroenterol Hepatol 12: A27-A28, 2014.

4. Fan NN, Yang J, Sun G, Lu ZS, Ling Hu EQ, Wang XD and Yang YS: Changes in the spectrum of gastric polyps in the Chinese population. World J Gastroenterol 21: 9758-9764, 2015.

5. Cao H, Wang B, Zhang Z, Zhang H and Qu R: Distribution trends of gastric polyps: An endoscopy database analysis of 24121 northern Chinese patients. J Gastroenterol Hepatol 27: 1175-1180, 2012.

6. Carmack SW, Genta RM, Schuler CM and Saboorian MH: The current spectrum of gastric polyps: A 1-year national study of over 120,000 patients. Am J Gastroenterol 104: 1524-1532, 2009.

7. Zheng E, Ni S, Yu Y, Wang Y, Weng X and Zheng L: Impact of gender and age on the occurrence of gastric polyps: Data analysis of 69575 southeastern Chinese patients. Turk J Gastroenterol 26: 474-479, 2015.

8. Buyukasik K, Sevinc MM, Gunduz UR, Ari A, Gurbulak B, Toros $\mathrm{AB}$ and Bektas $\mathrm{H}$ : Upper gastrointestinal tract polyps: What do we know about them? Asian Pac J Cancer Prev 16: 2999-3001, 2015.

9. Standards of Practice Committee; Lichtenstein DR, Jagannath S, Baron TH, Anderson MA, Banerjee S, Dominitz JA, Fanelli RD, Gan SI, Harrison ME, et al: Sedation and anesthesia in GI endoscopy. Gastrointest Endosc 68: 205-216, 2008.

10. ASGE Standards of Practice Committee; Evans JA, Chandrasekhara V, Chathadi KV, Decker GA, Early DS, Fisher DA, Foley K, Hwang JH, Jue TL, et al: The role of endoscopy in the management of premalignant and malignant conditions of the stomach. Gastrointest Endosc 82: 1-8, 2015.

11. Lin Y, Nie Y, Wang H, Li QQ and Li Y: Analysis of clinical pathological characteristics and pattern changes of 2643 gastric polyps in the past 15 years. Chin J Digest 4: 247-250, 2014. 